\title{
Ethical Considerations of Treating Patients Infected with Hepatitis C
}

\section{Virus}

\author{
Alireza Ebrahimi (iD ${ }^{1}$ and Sedigheh Ebrahimi (iD) ${ }^{2, *}$ \\ ${ }^{1}$ Student Research Committee, Shiraz University of Medical Sciences, Shiraz, Iran \\ ${ }^{2}$ Department of Medical Ethics, Shiraz University of Medical Sciences, Shiraz, Iran \\ "Corresponding author: Department of Medical Ethics, Shiraz University of Medical Sciences, Shiraz, Iran. Email: sedighebrahimi@gmail.com \\ Received 2020 August 09; Accepted 2020 October 17.
}

Keywords: Hepatitis C, Ethic, Barriers, Treatment

\section{Dear Editor,}

Hepatitis C virus (HCV) is a major cause of chronic hepatitis, which carries high mortality and morbidity (1). It's several decades that interferon-based regimens and direct-acting antiviral (DAAs) have improved the treatment of HCV (2). Although the World Health Organization (WHO) proposed a framework for eliminating HCV, several multiple-layered barriers may delay this goal (3). The barriers may be related to the patients (e.g. poor knowledge and asymptomatic infection), providers (e.g. treatment misconception), and health-care systems (e.g. high price and lack of strategies) (3).

To globally eliminate HCV infection by 2030 , as emphasized by the WHO, several ethical barriers should be considered. As noted by previous studies, these ethical considerations may deeply root in the higher prevalence of the disease among disadvantaged and vulnerable groups (e.g. prisoners, intravenous drug abusers, veterans, minority groups, and patients with psychiatric disorders) (4).

Moreover, the physicians' general attitudes toward the disease are poor and relatively negative, especially in lower-privileged countries, as it's believed that HCV cannot be treated (5). While the DAAs have greatly improved HCV-related diseases, these medications are not affordable for many people yet (USD93000 for a 12-week course) (6), which has caused several ethical dilemmas concerning the HCV treatment. On the other hand, those with comorbidities psychiatric diseases, and ongoing drug or alcohol abuse cannot be treated with antiviral drugs, an issue mentioned by several studies (7). The treating physicians might have justifications for regulating the availability of the antiviral therapies, including risk/benefit balance, patients' compliance, and treatment cost-effectiveness (4). However, limiting the HCV treatment to "good candidates" causes several ethical considerations such as autonomy, beneficence, nonmaleficence, justice, and respect for persons (4).

To respect the ethical considerations, while caring for patients with HCV, physicians should decide on treatment plans in the context of physician-patient relationships. The treatments should be available to all patients regardless of their social, psychological, and biological backgrounds. Moreover, the DAAs should be available to all patients that can be safely and effectively treated, even the most underprivileged and stigmatized patients. The core ethical dilemma regarding HCV care arises when the physicians try to balance the individuals' rights to access the treatments against communal benefits (8).

Such decisions should be taken on a case-by-case basis by providers while considering the history of the disease, its definite prevalence and course, availability of the cure, and the patients' compliance with medication therapy. Also, organizational, industrial, financial, and political factors may cause ethical considerations when making decisions regarding the treatment of HCV patients, which should be resolved $(5,8)$. Researchers should take reasonable steps to find a decision process for treating HCV patients that, while respecting medical ethics principles, reduces the burden of the disease.

\section{Footnotes}

Authors' Contribution: Study concept and design: SE. Drafting of the manuscript: AE. Critical revision of the manuscript for important intellectual content: SE. Study supervision: SE.

Conflict of Interests: The authors declare no conflict of interest regarding the publication of this paper. 
Funding/Support: None declared by the author.

\section{References}

1. Kasraian L, Hosseini S, Salehi Marzijarani M, Ebrahimi A, AshkaniEsfahani S. The prevalence of hepatitis $\mathrm{C}$ infection in blood donors: A meta-analysis and systematic review. Iran Red Crescent Med J. 2020;22(1). doi: 10.5812/ircmj.94998.

2. Roche B, Coilly A, Duclos-Vallee JC, Samuel D. The impact of treatment of hepatitis C with DAAs on the occurrence of HCC. Liver Int. 2018;38 Suppl 1:139-45. doi: 10.1111/liv.13659. [PubMed: 29427487].

3. Mendizabal M, Alonso C, Silva MO. Overcoming barriers to hepatitis C elimination. Frontline Gastroenterol. 2019;10(3):207-9. doi: 10.1136/flgastro-2018-101114. [PubMed: 31288259]. [PubMed Central: PMC6583574].

4. Geppert CM, Arora S. Ethical issues in the treatment of hepatitis C. Clin Gastroenterol Hepatol. 2005;3(10):937-44. doi: 10.1016/s15423565(05)00183-7. [PubMed: 16234037].
5. Behzadifar M, Azari S, Gorji HA, Martini M, Bragazzi NL. The hepatitis $\mathrm{C}$ virus in Iran: health policy, historical, ethical issues and future challenges. J Prev Med Hyg. 2020;61(1):E109-18. doi: 10.15167/24214248/jpmh2020.61.1.1438. [PubMed: 32490276]. [PubMed Central: PMC7225642].

6. Danta M, Ghinea N. The complex legal and ethical issues related to generic medications. Viral hepatitis: a case study. J Virus Erad 2017;3(2):77-81. [PubMed: 28435690]. [PubMed Central: PMC5384269]

7. Falck-Ytter Y, Kale H, Mullen KD, Sarbah SA, Sorescu L, McCullough AJ. Surprisingly small effect of antiviral treatment in patients with hepatitis C. Ann Intern Med. 2002;136(4):288-92. doi: 10.7326/0003-4819136-4-200202190-00008. [PubMed: 11848726].

8. Craxi L, Sacchini D, Refolo P, Minacori R, Daloiso V, Ricci G, et al. Prioritization of high-cost new drugs for HCV: making sustainability ethical. Eur Rev Med Pharmacol Sci. 2016;20(6):1044-51. [PubMed: 27049255] 